\title{
WATER DROPLET TRAJECTORIES IN A SPRINKLER SPRAY FLOW: THE CLASSIC VERSUS QUANTUM AND SINGLE VERSUS MULTI-DROPLET PICTURES
}

\author{
DANIELE DE WRACHIEN ${ }^{1}$, GIULIO LORENZINI ${ }^{2} \&$ STEFANO MAMBRETTI $^{3}$ \\ ${ }^{1}$ Department of Environmental and Agricultural Sciences, University of Milan, Italy. \\ ${ }^{2}$ Department of Industrial Engineering, University of Parma, Italy. \\ ${ }^{3}$ DIIAR, Politecnico di Milano, Italy.
}

\begin{abstract}
One of the most challenging modelling problems in modern engineering is that of a particle crossing a continuous phase (air). In sprinkler irrigation practice this may refer to a water droplet travelling in air from the nozzle to the ground. The challenge mainly refers to the difficulty in designing and solving the system of governing equations for that very complicate process, where many non-linearities occur when describing the relations and dependences among the parameters that rule the phenomenon. The problem becomes even more complicated when not just a single droplet alone is assessed but a multi-droplet system is accounted for. In addition to the inter-parameter dependencies, an inter-droplet reciprocal connection is also observed, mainly due to electrical interactions between the hydrogen and the oxygen atoms of the different water molecules. An alternative to traditional classic approaches to analyse water droplet dynamics in sprinkler irrigation has been recently proposed in the form of a quantum approach, but the whole classic-quantum and single-droplet versus multi-droplet alternatives need to be discussed and pinpointed which are among the main aims of the present paper which focuses on the theoretical part of the issue, thus highlighting the new perspectives of a deeper comprehension in the spray flow related phenomena.

Keywords: Classic and quantum mechanics, mathematical modelling, single-and multi-droplet systems, spray particles kinematics, sprinkler water droplets.
\end{abstract}

\section{INTRODUCTION}

Albeit the process of a liquid particle moving within a gaseous phase may describe many different technical applications, broadly investigated in many scientific sectors and from many different points of view, a complete, clear and generally applicable mathematical modelling is still far from having been achieved. Very recently Molle et al. [1] made an extremely useful experimental contribution in the field of irrigation, which will be of substantial usefulness for future investigations and modelling attempts. The results of the present paper were mainly reached by featuring the sprinkler irrigation context. The outcomes, if suitably adapted, may also apply to other fields and aims, such as pesticides distribution, heat removal or fire suppression, to name but a few. The fundamental problem is not just that of solving the equations ruling the development of the system and of the phenomenon but, upstream of that, it lies in the characterization of such equations. According to the authors of this paper, such hurdle can be attributed to incomplete understanding of the system-process evolution. This of course should not be taken as a form of underestimation of the analytical difficulties due to the mutual interrelationships between the parameters that govern the process, on the one hand, and the characteristics of the particles involved, on the other. In fact the main thematic scientific literature [2-6] tries to overcome such computational complication binding the solutions achieved to the specific case studies faced. In general, however, the kinematic analysis of sprinkler water droplets during their aerial path is devised adopting a Newtonian approach and considering a single-droplet 
system. A ballistic form of the same viewpoint, based on a Newtonian approach for a singledroplet system, was also proposed by the authors [7-9]. This approach will be described in the paper as a classic/single-droplet model. Coming again to the "not complete understanding of the system-process evolutions" quoted above, the issue has very recently led the authors of the present work to the belief that to fully comprehend and describe the phenomenon, another viewpoint could be considered: the quantum one [10]. The results which were arrived at [11] were in the form of the time-dependent Schroedinger's equation and of the scale relativity theory [12] written as a Riccati equation. The former, in particular, was written for single-droplet systems, seen as waves and material particles [13] and considering a Lagrangian or Eulerian description for both steady and transient states. The present paper, therefore, will treat further possibilities to study the kinematic behaviour of both single- and multi-droplet systems during their aerial path in/(according to) both the classic (i.e. Newtonian) and quantum approach.

\section{THE CLASSIC MECHANICAL PICTURE}

We do not wish to review the whole classic approach, as reported in the literature, but to explore the modelling possibilities in relation to the topic of the present paper. Anyway to further deepen the state-of-the-art, one could refer to other publications by De Wrachien and Lorenzini [7-9]. Some more information on spray kinematics modelling (mainly Lagrangian) both in sprinkler irrigation and in chemical sprays contexts are also available [3,14-16], while spray drift Lagrangian modelling is treated in Hewitt et al. [17], and in Bird et al. [18]. Recently the authors_[7-9] defined and validated (see also; [2,6]) the following simplified analytical model feasible to solve water droplets kinematics, based on the second principle of dynamics:

$$
\left\{\begin{array}{l}
m \ddot{x}=-k \dot{x}^{2} \\
m \ddot{y}=-k \dot{y}^{2}-n g
\end{array}\right.
$$

developed in terms of parametric equations:

$$
\begin{array}{r}
x(t)=\frac{m}{k} \ln \left(\frac{v_{0 x} k}{m} t+1\right) \quad \dot{x}(t)=\frac{m v_{0 x}}{m+k v_{0 x} t} \\
y(t)=h-\frac{m}{k} \ln \frac{\cos \left(\arctan \frac{\sqrt{\frac{k}{m}} v_{0 y}}{\sqrt{\frac{n}{m} g}}\right)}{\cos \left[\arctan \frac{\sqrt{\frac{k}{m}} v_{0 y}}{\sqrt{\frac{n}{m}}-t} \frac{\sqrt{k n g}}{m}\right)} \\
\dot{y}(t)=-\sqrt{\frac{n g}{k}} \tan \left[-\frac{\sqrt{n g k}}{m} t+\arctan \left(\sqrt{\frac{k}{n g}} v_{0 y}\right)\right]
\end{array}
$$


being: $f$ the friction factor according to Fanning [19]; $g$ the gravity; $h[\mathrm{~m}]$ the initial $y$ coordinate; $k=f \rho A / 2$ the friction coefficient; $m$ the particle mass; $n$ the droplet actual mass (buoyancy); $t$ the time; $v_{0 x}$ and $v_{0 y}$ the initial horizontal and vertical velocity components; $x, y, \dot{x}, \dot{y}, \ddot{x}, \ddot{y}$ the co-ordinates, velocities and accelerations along the horizontal and vertical axes, respectively. Being the model analytical, albeit simplified, it is applicable to a variety of problems but the more reliable results were obtained for high Reynolds numbers. Obviously, as mentioned above, the model presented is one of the possible ones which can describe a single-droplet system from a classic viewpoint: the choice was mainly due to the fact that such model is tightly related to the second law of dynamics, as previously mentioned. To complete the topic, anyway, one may in general face the kinematic analysis of a multi-droplet system (i.e. composed of $N$ droplets) from a classic viewpoint by means of the following analytical expression [20]:

$$
m_{k} \frac{d^{2} Q_{k}(t)}{d t^{2}}=-\nabla_{k} V \cdot Q(t)
$$

where $m_{k}(1 \leq k \leq N)$ is the $k$-th particle mass, $Q$ is the classic trajectory, $V$ is a potential function accounting for time dependence [20] and $\nabla_{k}$ is the 3-D gradient operator referred to the $k$-th particle.

\subsection{Validation of the dynamic model}

The validation of the classical procedure needs a quantitative approach to check how reliable the predictions are: this can be done introducing other authors' data in the model. The works chosen for these comparison purposes are Edling's [2] and Thompson et al.'s [6]. Their date set are reported in Table 1.

Comparisons of field measurements and theoretical values are presented in Tables 2, 3 and 4 in terms of travel distance (Tables 2 and 3 for Edling's and Thompson et al.'s cases respectively), and of time of flight (Table 4 for Thompson et al.'s cases only, since some data required for the computation were missing from Edling [2]). Table 2 shows reasonable agreement in two cases, but poor agreement with a droplet diameter of $0.5 \mathrm{~mm}$. Tables 3 and 4 present a comparative analysis on the basis of Thompson et al.'s [6] data in terms of

Table 1: Reference data set for the comparative analyses of results.

\begin{tabular}{llc}
\hline & Edling [2] & Thompson et al. $[6]$ \\
\hline Flow rate exiting from the sprinkler $\left(\mathrm{dm}^{3} / \mathrm{s}\right)$ & $1.4 \times 10^{-4}$ & $5.5 \times 10^{-4}$ \\
Nozzle diameter $(\mathrm{mm})$ & 3.96 & 4.76 \\
Jet inclination with respect to horizontal $\left({ }^{\circ}\right)$ & 0 & 25 \\
& 10 & \\
Nozzle height $(\mathrm{m})$ & -10 & 4.5 \\
& 1.22 & \\
Air temperature $\left({ }^{\circ} \mathrm{C}\right)$ & 2.44 & 38 \\
Wind & 3.66 & No \\
\hline
\end{tabular}


Table 2: Travel distance of sprinkler droplets: Edling's [2] data vs Lorenzini's [8].

\begin{tabular}{|c|c|c|c|c|c|c|c|}
\hline \multirow{3}{*}{$\begin{array}{l}\text { Nozzle } \\
\text { height } \\
\text { (m) }\end{array}$} & \multirow{3}{*}{$\begin{array}{l}\text { Jet } \\
\text { inclin. } \\
\text { (deg) }\end{array}$} & \multicolumn{6}{|c|}{ Droplet diameter (m) } \\
\hline & & \multicolumn{2}{|c|}{$0.5 \times 10^{-3}$} & \multicolumn{2}{|c|}{$1.5 \times 10^{-3}$} & \multicolumn{2}{|c|}{$2.5 \times 10^{-3}$} \\
\hline & & $\begin{array}{c}\text { Edling } \\
\text { [2] }\end{array}$ & $\begin{array}{l}\text { Lorenzini } \\
{[8]}\end{array}$ & $\begin{array}{c}\text { Edling } \\
{[2]}\end{array}$ & $\begin{array}{c}\text { Lorenzini } \\
{[8]}\end{array}$ & $\begin{array}{c}\text { Edling } \\
\text { [2] }\end{array}$ & $\begin{array}{c}\text { Lorenzini } \\
{[8]}\end{array}$ \\
\hline \multirow[t]{3}{*}{1.22} & 10 & 1.53 & 2.11 & 4.04 & 4.29 & 5.08 & 5.22 \\
\hline & 0 & 1.52 & 1.77 & 3.55 & 3.38 & 4.19 & 3.98 \\
\hline & -10 & 1.46 & 1.35 & 2.91 & 2.48 & 3.22 & 2.85 \\
\hline \multirow[t]{3}{*}{2.44} & 10 & 1.55 & 2.20 & 4.62 & 4.81 & 6.00 & 6.00 \\
\hline & 0 & 1.55 & 1.92 & 4.31 & 4.08 & 5.37 & 5.00 \\
\hline & -10 & 1.50 & 1.52 & 3.86 & 3.27 & 4.57 & 3.96 \\
\hline \multirow[t]{3}{*}{3.66} & 10 & 1.55 & 2.22 & 4.95 & 5.11 & 6.60 & 6.50 \\
\hline & 0 & 1.55 & 1.96 & 4.73 & 4.47 & 6.10 & 5.62 \\
\hline & -10 & 1.50 & 1.57 & 4.36 & 3.71 & 5.41 & 4.64 \\
\hline
\end{tabular}

Table 3: Travel distance of sprinkler droplets:

Thompson et al.'s [6] data vs Lorenzini's [8].

\begin{tabular}{lcc}
\hline Droplet diameter $(\mathrm{mm})$ & Thompson et al. [6] & Lorenzini [8] \\
\hline 0.3 & 1.30 & 2.73 \\
0.9 & 5.22 & 6.77 \\
1.8 & 10.00 & 11.56 \\
3.0 & 13.48 & 16.66 \\
5.1 & 17.83 & 23.59 \\
\hline
\end{tabular}

Table 4: Time of flight of sprinkler droplets:

Thompson et al.'s [6] data vs Lorenzini's [8].

\begin{tabular}{lcc}
\hline Droplet diameter $(\mathrm{mm})$ & Thompson et al. $[6]$ & Lorenzini [8] \\
\hline 0.3 & 2.63 & 0.84 \\
0.9 & 1.54 & 1.35 \\
1.8 & 1.63 & 1.73 \\
3.0 & 1.75 & 2.00 \\
5.1 & 1.84 & 2.26 \\
\hline
\end{tabular}

travel distance and time of flight, respectively. A difference can be seen with a droplet diameter of $0.3 \mathrm{~mm}$. This is due to the flow description adopted in Thompson et al. [6] for smaller droplets, which was not shared in the present approach with regard to the diffusion effects. The other data, particularly those referring to the range of the intermediate droplet diameters, instead show reasonable agreement both in the values obtained and in the trends determined. 
Facing a comparative approach, it can be stated that the model here defined proves to be kinematically reliable in its predictions from a qualitative and quantitative points of view, particularly when droplets having a "not too small" diameter are considered. This, being the model defined by neglecting most of the parameter typically introduced in the others, can be considered as a first relevant result. The comparisons performed with Thompson et al.'s data show that when the droplet gets close to a condition of Stokes' flow law, the model provides less accurate results. This is the limit to the model and it somehow defines the field of acceptability of the method presented here. The model becomes weaker when it moves away from Newton's flow law because of the approximation used to define $k$ in the other two flow patterns. The dependence of the results on the flow state criterion of small diameter can easily explain the different results obtained for the smallest droplets in the present work and in Thompson et al. [6].

\section{THE QUANTUM MECHANICAL PICTURE}

\subsection{Quantum mechanics for a single particle}

The discrepancies between a classic vs. quantum description, on the one hand, and between a single-droplet vs. multi-droplet one, on the other, may be highlighted examining the following expressions for single- and multi-droplet systems (respectively) as compared to those in the previous section of this paper [11,20]:

$$
\begin{gathered}
m \frac{d^{2} Q(t)}{d t^{2}}=F(t) \\
m_{k} \frac{d^{2} Q_{k}(t)}{d t^{2}}=\nabla_{k}\left(V \mid V_{q u}^{\psi_{t}}\right) Q(t)
\end{gathered}
$$

where $F$ is the force, $m$ the particle mass, $V_{q u}^{\psi_{t}}=-\sum_{j=1}^{N} \frac{\hbar^{2}}{2 m_{j}} \frac{\nabla_{j}^{2}|\psi|}{|\psi|}(1 \leq j<k \leq N)$ the quantum potential, $\hbar$ the Dirac constant. Comparing eqn (5) with (3), the first useful consideration is that, if the quantum potential assumes a value which is in the vicinity of zero, then the quantum and classic kinematic pictures tend to coincide. But as a quantum viewpoint presumes that the "object" evaluated is not just a material particle but also a wave, then for each element of a multi-droplet system one may write the time-dependent Schroedinger's equation as:

$$
D^{2} \nabla^{2} \psi(\vec{x}, t)-\frac{1}{2} \cdot m \cdot V(\vec{x}, t) \cdot \psi(\vec{x}, t)=-i \cdot D \cdot\left(\frac{\partial}{\partial t}\right) \cdot \psi(\vec{x}, t)
$$

where $D$ is the diffusion coefficient, $\psi(\vec{x}, t)=R(\vec{x}, t) \cdot \exp [S(\vec{x}, t)], R$ is the wave amplitude, $S$ is the wave phase. Equation (6) can be re-written in the form of continuity and Euler-type "quantum fluid-dynamic equations", respectively [11,21,22]:

$$
\begin{gathered}
\frac{\partial}{\partial t} \rho(\vec{x}, t)+\nabla(\rho(\vec{x}, t) \cdot \vec{x}(\vec{x}, t))=0 \\
\frac{\partial}{\partial t} \vec{v}(\vec{x}, t) \equiv\left[\frac{\partial}{\partial t}+\vec{v}(\vec{x}, t) \cdot \nabla\right] \vec{v}(\vec{x}, t)=-\frac{1}{m} \cdot \nabla[V(\vec{x}, t)+Q(\vec{x}, t)]
\end{gathered}
$$


( $\rho$ is density, $\vec{v}$ is velocity, $V$ is the classic potential, $Q$ is the quantum potential). Equation (6) can be re-worked by means of Nottale's scale relativity theory [12] using a probability density function for a semi-infinite domain [23] for writing the second law of dynamics in the complex field ( $u$ is a scalar potential, $W$ is a complex velocity):

$$
-\nabla u=m \cdot \frac{\partial}{d t} W
$$

Dividing the real and imaginary parts in eqn (9) ( $U$ is the imaginary part of $W$ ) one gets:

$$
\left\{\begin{array}{l}
-D \cdot \Delta U-(U \cdot \nabla) U=-\nabla u \\
\frac{\partial}{\partial t} U=0
\end{array}\right.
$$

which (first equation) may be re-written for a 1-D path as a Riccati equation [24], being $c$ a constant and $y(x)$ an arbitrary function of $x$ :

$$
\begin{aligned}
& \frac{d}{d x} U(x)=-\frac{m}{\hbar} \cdot U^{2}(x)+\frac{2}{\hbar} \cdot(u(x)-c \cdot m) \\
& \frac{d^{2}}{d x^{2}} y(x)-\frac{2 \cdot m}{\hbar^{2}} \cdot(u(x)-c \cdot m) \cdot y(x)=0
\end{aligned}
$$

Both are very powerful tools as they allow for quantum particles computations avoiding the time-dependent Schroedinger's equation, even if it just for 1-D domains, which is useful in particular cases as for instance a droplet vertical downfall.

\subsection{Quantum mechanics for many-particle systems}

Considering multi-droplet systems, the time-dependent Schroedinger's equation needs to be suitably re-written, provided that water has a V-shaped molecule resulting in a magnetic field due to the electric potential between oxygen and hydrogen. This results in [21]:

$$
\left[\frac{1}{2} \sum_{j}\left(-2 i D \nabla_{j}-\vec{K}\left(\overrightarrow{x_{j}}, t\right)\right)^{2}+\frac{1}{m} V\left(\overrightarrow{x^{N}}, t\right)\right] \psi\left(\overrightarrow{x^{N}}, t\right)=2 i D \frac{\partial \psi\left(\overrightarrow{x^{N}}, t\right)}{\partial t}
$$

being $V\left(\overrightarrow{x^{N}}, t\right)$ the electric potential, $\phi\left(\overrightarrow{x_{j}}, t\right)$ the external time-dependent scalar potential, $\overrightarrow{x^{N}}$ the $N$-particle coordinates. This may be transformed similarly to single-droplet systems as:

$$
\psi\left(\overrightarrow{x^{N}}, t\right)=R\left(\overrightarrow{x^{N}}, t\right) \exp \left[S\left(\overrightarrow{x^{N}}, t\right)\right]
$$

then giving the continuity equation:

$$
\frac{\partial \rho^{N}\left(\overrightarrow{x^{N}}, t\right)}{\partial t}+\sum_{k=1}^{N} \nabla k \overrightarrow{\mathbf{J}_{k}}\left(\overrightarrow{x^{N}}, t\right)=0
$$


and the Euler-type equation:

$$
\begin{aligned}
& \frac{\partial \overrightarrow{v_{k}}\left(\overrightarrow{x^{N}}, t\right)}{\partial t}+\sum_{j}\left(\overrightarrow{v_{j}}\left(\overrightarrow{x^{N}}, t\right) \nabla k\right) \overrightarrow{v_{j}}\left(\overrightarrow{x^{N}}, t\right)+\sum_{j}\left(1-\delta_{j k}\right) \overrightarrow{v_{j}}\left(\overrightarrow{x^{N}}, t\right) \times\left(\nabla_{k} \times \overrightarrow{v_{j}}\left(\overrightarrow{x^{N}}, t\right)\right)= \\
& =-\left(e \vec{E}\left(\overrightarrow{x_{k}}, t\right)+\frac{e}{c} \overrightarrow{v_{k}}\left(\overrightarrow{x^{N}}, t\right) \times \vec{B}\left(\overrightarrow{x_{k}}, t\right)\right)-\frac{1}{m} \nabla\left[V_{0}\left(\overrightarrow{x^{N}}, t\right)+U\left(\overrightarrow{x^{N}}, t\right)+Q\left(\overrightarrow{x^{N}}, t\right)\right]
\end{aligned}
$$

where $\nabla_{k}$ is the gradient operator related to the coordinate $\overrightarrow{x_{k}}$ of the $k$-th particle; $\rho^{N}\left(\overrightarrow{x^{N}}, t\right)=R^{2}\left(\overrightarrow{x^{N}}, t\right)$ the $N$-particle density; $\overrightarrow{J_{k}}\left(\overrightarrow{x^{N}}, t\right)=\rho^{N}\left(\overrightarrow{x^{N}}, t\right) \cdot \overrightarrow{v_{k}}\left(\overrightarrow{x^{N}}, t\right)$ the fluid current density; $\overrightarrow{v_{k}}\left(\overrightarrow{x^{N}}, t\right)=\frac{\hbar}{m} \cdot \nabla_{k} S\left(\overrightarrow{x^{N}}, t\right)-\frac{e}{m c} \cdot \vec{A}\left(\overrightarrow{x^{N}}, t\right)$ the velocity field of the $h$-th particle; $\vec{E}\left(\overrightarrow{x_{k}}, t\right)=-\nabla \phi\left(\overrightarrow{x_{j}}, t\right)-\frac{1}{c} \cdot \frac{\partial \vec{A}\left(\overrightarrow{x^{N}}, t\right)}{\partial t}$ the external electric field; $\vec{B}\left(\overrightarrow{x_{k}}, t\right)=\operatorname{curl} \vec{A}\left(\overrightarrow{x^{N}}, t\right)$ the external magnetic field. The first integrations of eqns (15) and (16) were carried out by Madelung [25], and the work was successively extended by Bohm [26,27].

\section{THE DYNAMICAL AND NUMERICAL APPROXIMATIONS}

In any case an analytical "closed form" solution of the equations describing the quantum kinematics of particles is obviously extremely difficult and even the most advanced techniques often fail to achieve such purpose, albeit in the years to come this attempt will not be abandoned. This is why, recently, different forms of approximation have been introduced to treat the "quantum fluid-dynamic equations": among those, literature reports numerical and dynamical approximations [28-30], which are both currently being developed. The formers may rely on Eulerian, Lagrangian or arbitrary Lagrangian-Eulerian descriptions, all characterised by advantages and disadvantages. Lagrangian descriptions are easier in the form through which they write down the equations, as the grid moves with the particle and follows its evolution; but they become difficult to handle as, step after step, the grid becomes non-uniform with problems in the accuracy of the flow solution. Eulerian descriptions are complicate at the beginning of the simulation, due to an increased analytical complication, but prove to be more practical afterwards as the grid does not change with time. A uniform grid following the flow evolution is instead met in the arbitrary Lagrangian-Eulerian descriptions, also adopted in some computational fluid dynamics codes. The dynamical approximations do not rely in a mathematically-simplified description of the problem but in a physically-simplified one by superimposing some particular conditions (e.g. incompressible flow) or neglecting some other characteristics considered not so relevant to the whole picture. Obviously it would not be inconceivable to imagine a mixed numerical-dynamical approximation approach and we feel that on this aspect research will invest a part of its future resources: in relation to this challenge one should highlight that quantum trajectories can be treated quite similarly to the classic ones when considering, for the particles treated, the suitable relations among the dynamic and the potential part of the problem.

\section{CONCLUSIONS}

Describing in an analytically and physically correct way, the phenomenon of a water droplet travelling from the exit of a sprinkle nozzle down to the ground is an extremely difficult task, as many studies performed in the last decades have broadly demonstrated, trying to find a 
solution via different means and approaches. The present investigation starts from a recent hypothesis made by the same authors of this paper: a water droplet could be treated as a quantum object, characterised both by material particle and wave properties. Thus the time-dependent Schroedinger's equation may be employed to study the process and a parallel classic-quantum description may be achieved, both for single-droplet and for multi-droplet systems. The latter systems are not only affected by the usual fluid-dynamic parameters but the mutual repulsions and attractions between particles are to be accounted for, in the form of electric-magnetic potentials bound to the molecular structure of water: this allows one to re-write the time-dependent Schroedinger's equation and the so-called "quantum fluiddynamic equations" in a novel and more complete form. In addition, the paper provides a general overview of the numerical and dynamical approximations currently available to treat the systems of equations arrived at. Future studies will deepen the novel modelling approach suggested to make it more and more suitable for practical applications.

\section{REFERENCES}

[1] Molle, B., Tomas, S., Hendawi, M. \& Granier, J., Evaporation and wind drift losses during sprinkler irrigation influenced by droplet size distribution. Irrigation and Drainage, 61(2), pp. 240-250, 2012. doi: http://dx.doi.org/10.1002/ird.648

[2] Edling, R.J., Kinetic energy, evaporation and wind drift of droplets from low pressure irrigation nozzles. Transactions of the ASAE, 28(5), pp. 1543-1550, 1985.

[3] Keller, J. \& Bliesner, R.D., Sprinkler and Trickle irrigation, Van Nostrand Reinhold: New York, 1990. doi: http://dx.doi.org/10.1007/978-1-4757-1425-8

[4] Kincaid, D.C. \& Longley, T.S., A water droplet evaporation and temperature model. Transactions of the ASAE, 32(2), 457-463, 1989.

[5] Kinzer, G.D. \& Gunn, R., The evaporation, temperature and thermal relaxation-time of freely falling waterdrops. Journal of Meteorology, 8(2), 71-83, 1951. doi: http://dx.doi. org/10.1175/1520-0469(1951)008<0071:TETATR >2.0.CO;2

[6] Thompson, A.L., Gilley, J.R. \& Norman, J.M.A., Sprinkler water droplet evaporation and plant canopy model: II. Model applications. Transactions of the ASAE, 36(3), 743-750, 1993.

[7] De Wrachien, D. \& Lorenzini, G., Modelling jet flow and losses in sprinkler irrigation: overview and perspective of a new approach. Biosystems Engineering, 94(2), pp. 297-309, 2006. doi: http://dx.doi.org/10.1016/j.biosystemseng.2006.02.019

[8] Lorenzini, G., Simplified modelling of sprinkler droplet dynamics. Biosystems Engineering, 87(1), pp. 1-11, 2004. doi: http://dx.doi.org/10.1016/j.biosystemseng.2003. $\underline{08.015}$

[9] Lorenzini, G., Water droplet dynamics and evaporation in an irrigation spray. Transactions of the ASABE, 49(2), pp. 545-549, 2006.

[10] Dirac, P.A., Quantized singularities in the electromagnetic field. Proceedings of the Royal Society. A133, pp. 1-60, 1931.

[11] De Wrachien, D., Lorenzini, G. \& Mambretti, S., Water droplet trajectories in an irrigation spray: the classic and quantum mechanical pictures. 40th International Symposium on Agricultural Engineering, Opatija (Croatia), pp. 85-96, 2012.

[12] Nottale, L., The theory of scale relativity. International Journal of Modern Physics, 7(20), pp. 4899-4935, 1992. doi: http://dx.doi.org/10.1142/S0217751X92002222

[13] Goldstein, S., Tumulka, R. \& Zanghi, N., Bohmian trajectories as the foundation of quantum mechanics. Quantum Trajectories, ed. Chattaraj, CRC Press, Taylor and Francis Group, pp. 1-15, 2011. 
[14] Teske, M.E., Hermansky, C.G. \& Riley, C.M., Evaporation rates of agricultural spray material at low relative wind speeds. Atomization and Spray, 8, pp. 471-478, 1998a.

[15] Teske, M.E., Thistle, H.W. \& Eav, B., New ways to predict aerial spray deposition and drift. Journal of Forestry, 96(6), pp. 25-31, 1998 b.

[16] Teske, M.E. \& Ice, G.G., A one-dimensional model for aerial spray assessment in forest streams. Journal of Forestry, 100(3), pp. 40-45, 2002.

[17] Hewitt, A.J., Johnson, D.A., Fish, J.D., Hermansky, C.G. \& Valcore, D.L., Development of spray drift task force database for aerial applications. Environmental Toxicology and Chemistry, 21(3), pp. 648-658, 2002. doi: http://dx.doi.org/10.1002/etc.5620210326

[18] Bird, S.L., Perry, S.G., Ray, S.L. \& Teske, M.E., Evaluation of the AGDISP aerial spray algorithms in the AgDRIFT model. Environmental Toxicology and Chemistry, 21(3), pp. 672-681, 2002. doi: http://dx.doi.org/10.1002/etc.5620210328

[19] Bird, R.B., Steward W.E. \& Lighfoot, E.N., Transport Phenomena, Wiley and Sons: New York, 1960.

[20] Lopreore, C.L. \& Wyatt, R.E., Quantum wave packet dynamics with trajectories. Physical Review Letters, 82, pp. 5190-5193, 1999. doi: http://dx.doi.org/10.1103/ PhysRevLett.82.5190

[21] Ghosh, S.K., Quantum fluid dynamics within the framework of density functional theory. Quantum Trajectories, ed. Chattaraj, CRC Press, Taylor and Francis Group, pp. 183-195, 2011.

[22] Wyatt, R.E., Quantum Dynamics with Trajectories. Introduction to Quantum Dynamics, Springer, pp. 1-405, 2005.

[23] Hermann, R.P., Numerical simulation of a quantum particle in a box. Journal of Physics 1: Mathematical and General, 30(11), 3967-3975, 1997. doi: http://dx.doi. org/10.1088/0305-4470/30/11/023

[24] Al-Rashid, S.N.T., Habeeb, M.A. \& Amed, K.A., Application of the scale relativity $(\mathrm{ScR})$ theory to the problem of a particle in a finite one-dimensional square well (FODSW) potential. Journal of Quantum Information Science, 1, pp. 7-17, 2011. doi: http://dx.doi.org/10.4236/jqis.2011.11002

[25] Madelung, E., Eine anschauliche Deutung der Gleichung von Schroedinger. Naturwissenschaften, 14(45), pp. 1004-1004, 1926. doi: http://dx.doi.org/10.1007/BF01504657

[26] Bohm, D., A suggested interpretation of the quantum theory in terms of "hidden" variables - I. Physical Review, 85(2), 166-179, 1952a. doi: http://dx.doi.org/10.1103/ PhysRev.85.166

[27] Bohm, D., A suggested interpretation of the quantum theory in terms of "hidden" variables - II. Physical Review, 85(2), 180-193, 1952b. doi: http://dx.doi.org/10.1103/ PhysRev.85.180

[28] Kendrick, B.K., Direct numerical solution of the quantum hydrodynamic equation of motion. Quantum Trajectories, ed. Chattaraj, CRC Press, Taylor and Francis Group, pp. 325-344, 2011.

[29] Holland, P., Quantum field dynamics from trajectories. Quantum Trajectories, ed. Chattaraj, CRC Press, Taylor and Francis Group, pp. 73-86, 2011.

[30] Lorenzini, G., Conti, A. \& De Wrachien, D., Computational fluid dynamics (CFD) picture of water droplet evaporation in air. Irrigation \& Drainage Systems Engineering, in press, 2012. doi: http://dx.doi.org/10.4172/2168-9768.1000101 